Int. J. Electrochem. Sci., 16 (2021) Article ID: 21084

International Journal of

ELECTROCHEMICAL

SCIENCE

www.electrochemsci.org

\title{
Ultrasensitive Determination of Dopamine and Uric Acid Based on Gold Nanoparticles@ Carbon Dots/Electrochemical Reduced Graphene Oxide modified glassy carbon electrode
}

\author{
Muyi Li, Xiang Yang, Wenjun Liu, Huixian Wang, Hongmei Jiang ${ }^{*}$, Xiaoying Liu* \\ School of Chemistry and Materials Science, Hunan Agricultural University, Changsha 410128, P.R. \\ China. \\ *E-mail: jhmndcn@hunau.edu.cn (H. Jiang), xyliu@hunau.edu.cn, xiaoyingliu@126.com (X. Liu)
}

doi: $10.20964 / 2021.08 .04$

Received: 14 March 2021 / Accepted: 13 May 2021 / Published: 30 June 2021

\begin{abstract}
An ultrasensitive electrochemical sensing platform based on a glassy carbon electrode (GCE) modified with electrochemically reduced graphene oxide (erGO) and gold nanoparticles@carbon dots (GNPs@CDs) was developed. The hydroxyl-rich CDs were electrochemically synthesized, and the GNPs@CDs were obtained by reducing chloroauric acid with hydroxyl-rich CDs. The electrochemical behavior of the fabricated electrode, GNPs@CDs/erGO/GCE, was explored by cyclic voltammetry and electrochemical impedance spectroscopy. The electrochemical performance of the electrode demonstrated a remarkable enhancement in the peak current toward oxidation of dopamine (DA) and uric acid (UA) with a peak potential separation of $0.15 \mathrm{~V}$ in the presence of ascorbic acid (AA). Under optimized conditions, the simultaneous determination of DA and UA was investigated using differential pulse voltammetry (DPV) resulting in a linear response in the concentration ranges of $0.01-5.0 \mu \mathrm{M}$ and 5.0-20.0 $\mu \mathrm{M}$ for DA and of $0.1-20.0 \mu \mathrm{M}$ and $20.0-50.0 \mu \mathrm{M}$ for UA and in a sensitivity of 11.96 and $6.48 \mu \mathrm{A} \mu \mathrm{M}^{-1}$, respectively. The prepared electrode also demonstrated long-term stability and good reproducibility and was successfully used for the determination of DA and UA in human serum samples with favorable recoveries.
\end{abstract}

Keywords: GNPs@CDs/erGO nanocomposite; electrochemical synthesis; simultaneous determination; dopamine; uric acid.

\section{$\underline{\text { FULL TEXT }}$}

(C) 2021 The Authors. Published by ESG (www.electrochemsci.org). This article is an open access article distributed under the terms and conditions of the Creative Commons Attribution license (http://creativecommons.org/licenses/by/4.0/). 